\title{
Algunas consideraciones éticas alrededor de las publicaciones médicas
}

\author{
Some ethical considerations about medical \\ journals
}

\author{
Eugenio Matijasevic • Bogotá, D.C. (Colombia)
}

La ética, de acuerdo con el Diccionario de la Real Academia, es el conjunto de normas morales que rigen la conducta de la persona en cualquier ámbito de la vida. También es, en otra de sus acepciones, la parte de la filosofía que trata del bien y del fundamento de sus valores (1).

El término ética en castellano se originó en la palabra latina eth̆cus, la cual proviene,

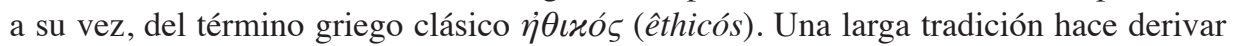
êthicós de $\eta \theta o \varsigma$ (ethos) que quiere decir costumbre, pero Joan Corominas, sin duda el más importante de los etimologistas del castellano, considera que proviene de un término afín, êthos, que no quiere decir precisamente costumbre sino carácter, manera de ser (2). El propio Aristóteles, el primero en utilizar el término j̇ $\theta \iota x o ́ \varsigma$ con el sentido que aún le damos en la actualidad parece estar de su lado cuando afirma en la Etica Nicomaquea que la virtud moral toma su nombre $\dot{\eta} \theta \iota x \eta \dot{~(e ̂ t h i k e ̂) ~ a ~ p a r t i r ~ d e ~ u n a ~ l i g e r a ~ a l t e r a c i o ́ n ~ d e l ~}$ término $\dot{\eta} \theta o \varsigma$ (êthos) que significa hábito, costumbre (3).

De acuerdo con Aristóteles, la relación entre hábito y carácter no es solo lingüística, en la medida en que las virtudes morales que conforman nuestro carácter no nos vienen dadas en nuestra $\phi v ́ \sigma \iota \varsigma$ (physis: naturaleza), no forman parte de nuestros dones naturales, sino que las debemos crear, cultivar y acrecentar mediante la educación y la práctica habitual. En este sentido, continúa Aristóteles, el hábito es crucial en la formación del carácter, pues es realizando actos valientes que nos volvemos valientes y realizando acciones justas que nos volvemos justos. No es estudiando qué es la justicia o estudiando qué es el valor que nos tornamos justos o valientes, es realizando acciones justas y valientes, habituándonos a ellas, que nuestro carácter será el de personas justas y valientes.

De hecho, ni la Ética Nicomaquea ni la Ética Eudemia de Aristóteles se leen como un tratado de antropología sobre los hábitos y costumbres de los griegos, sino como una indagación para responder a la pregunta sobre cómo deberían vivir los griegos para vivir mejor y alcanzar la $\varepsilon v \delta \alpha \iota \iota о v \iota \alpha$ [eudaimonía: felicidad, pero no en el sentido de placer, sino de florecimiento, de fructificación (4)] mediante el pleno ejercicio de sus virtudes

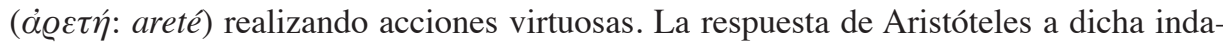
gación fue tan metódica, general y contundente que no sólo fue útil para los griegos de entonces, aún hoy, veintitrés siglos después, todos los seres humanos seguimos derivando profundas enseñanzas de su forma de dar respuesta a las preguntas ¿qué es una vida buena? y ¿cómo debemos vivir?.

Ahora bien, la ética para Aristóteles no era una ciencia teórica, como sus tratados de lógica o de metafísica, sino una ciencia práctica, al igual que el tratado que dedicó al buen gobierno de una $\pi o ́ \lambda \iota \varsigma$ (polis: ciudad), La Política: ambas, la ética y la política, eran para él ciencias en las que el aprendizaje teórico pasaba a un segundo plano frente a la necesidad de poner en práctica el conocimiento que aportaban, eran ciencias en las que el objeto de su estudio no era meramente llevar a cabo una investigación que diera lugar a una descripción de la vida buena y del buen gobierno, por el contrario, el objeto de su estudio era vivir bien y participar de la mejor manera posible en el buen gobierno de la
Eugenio Matijasevic, MD FACP: Editor General Acta Médica Colombiana. Bogotá, D.C. (Colombia).

E-mail: eugenio.matijasevic@gmail.com 
ciudad. El objetivo de la ética no es saber o describir qué es vivir bien, sino vivir bien obrando bien.

Con frecuencia en castellano intercambiamos los términos "ética" y "moral", aunque muchos autores intentan establecer diferencias entre ambos. Quienes opinan que se trata del mismo término aducen que así ha sido desde que Cicerón tradujo el adjetivo griego $\dot{\eta} \theta$ เxós por el término latino moralis (5). No es el momento de entrar en esa discusión, pero prefiero utilizar el término ética en lugar de moral en la medida en que en el castellano actual el término moral tiene otras múltiples acepciones con sentidos que lo alejan del sentido prácticamente unívoco que tiene el término ética. En efecto, en el Diccionario de la Real Academia el término "moral" posee ocho acepciones diferentes, entre ellas una muy afín a la definición que de ética trae el propio diccionario: "doctrina del obrar humano que pretende regular de manera normativa el valor de las reglas de conducta y los deberes que estas implican", al lado de otras que, en realidad, no tienen nada que ver con la ética, como por ejemplo: "en actividades que implican confrontación o esfuerzo intenso, confianza en el éxito" (6). Al respecto, dice Corominas, el término castellano "moral", aunque se deriva del latín morālis, derivado a su vez del latín $m \bar{o} s, m o ̄ r i s$, que significa deseo, capricho, uso, costumbre, manera de vivir, se usa también en otros sentidos, como el de "voluntad de lucha de las tropas", sentido que parece ser un galicismo (7).

Independientemente de su etimología, la definición del término ética gira siempre alrededor de la idea de cómo debemos obrar o de cómo debemos vivir la vida, que fue el sentido que originalmente le confirió Aristóteles. Sin embargo no todos los estudiosos del asunto están de acuerdo con él en que sea la vida buena, a través de la virtud, el parámetro que permita fijar una respuesta correcta a la pregunta de cómo debemos vivir la vida. De hecho, existen tantas definiciones de ética como escuelas filosóficas o escuelas de ética.

Ética es, para Thomas Scanlon, lo que nos debemos los unos a los otros [What We Owe to Each Other (8)] y, aunque con sólo decirlo ya se está adhiriendo a una escuela, el contractualismo, su definición es tan amplia en este caso que muy pocos eticistas, independientemente de la escuela a la que adhieran, podrían objetar que sea ese, en la práctica, como quería Aristóteles, el objeto de la ética.

Obviamente no fue Scanlon el primero en aproximarse de esta manera a la ética. Bien mirada, su frase trae reminiscencias de todos cuantos dieron a lo largo de la historia una definición de la denominada regla de oro de la ética, o regla de la reciprocidad, que ha sido enunciada una y mil veces, de mil diversas maneras, a lo largo de la historia y a todo lo ancho de la geografía de nuestro planeta (9), pero que puede enunciarse aún en los sencillos términos que lo hiciera Confucio cuando Zigong le preguntó si existía una única palabra que pudiera servirnos como guía de conducta a lo largo de la vida. El Maestro dijo: "Quizás es la palabra shu: no impongas a otros lo que tú mismo no deseas que otros te impongan a ti" (10). El significado de $s h u$ se encuentra al comienzo de las Analectas. Confucio le dijo al Maestro Zeng: "mi camino tiene un hilo que corre a través de él" y, después que Confucio se fue, los discípulos le preguntaron al Maestro Zeng qué había querido decir. El Maestro Zeng respondió: "El camino del Maestro consiste en hacer lo mejor para realizar su propia humanidad (zhong), y tratar a los demás con la conciencia de que también ellos están plenos de humanidad $(s h u)$ " (11). Hacia la misma época, aunque la fecha es más difícil de precisar, en el Mahabharata aparece una declaración de la regla de oro muy similar a la de Confucio: "Un hombre no debe actuar hacia otros de un modo que no le gustaría que actuaran hacia él. Conociendo aquello que no le agrada para si mismo, no debería actuar hacia otros de esa manera [...]. Actúa hacia los demás de manera que estés preparado para tolerarlo tú mismo" (12).

El enunciado de la regla de oro establece que nuestras acciones con respecto a los demás deben ser tales que aceptaríamos sin dificultad la reciprocidad de los demás: que ellos actuaran de la misma manera con respecto a nosotros. La reciprocidad es en este caso indispensable: acepto que actúen conmigo como yo he actuado con ellos. Pero dicha reciprocidad no puede, desde el punto de vista lógico, darse al revés: actúo con ellos como me gustaría que actúen conmigo. En este sentido tiene razón George Bernard Shaw cuando afirma, en su estilo iconoclasta y burlón: Do not do unto others as you would that they should do unto you. Their tastes may not be the same (13) (no hagas a otros lo que te gustaría que te hicieran a ti. Sus gustos pueden no ser los mismos). El enunciado de la regla de oro de Jesús de Nazareth carece de esta dificultad, pues enuncia la regla de oro sin utilizar una formulación prohibitiva (no aparece "no impongas" o "no debe actuar"), simplemente establece, en cada uno de los evangelios sinópticos: "Ama a tu prójimo como a ti mismo" [Mateo 22:39 (14), Marcos 12:31 (15), Lucas 10:27 (16)].

La manera en que Scanlon enuncia la regla de oro sigue esta misma línea de no prohibir sino de propender por una manera de relacionarnos claramente afirmativa: "lo que nos debemos los unos a los otros". Desde esta perspectiva la ética no sería una fe religiosa ni se adscribiría a ninguna fe religiosa (estaría abierta a todas las creencias religiosas), no sería tampoco una preceptiva (una lista de normas y preceptos), ni es una ciencia (en el sentido de un cuerpo de conocimientos teóricos), sería, más bien, un método para resolver en la práctica conflictos éticos, es decir, conflictos relacionados con lo que nos debemos los unos a los otros (17). Sólo cuando la ética falla (mejor dicho, cuando nosotros fallamos en nuestros deberes éticos de cuanto nos debemos los unos a los otros) es cuando comienzan a intervenir los preceptos normativos y las leyes.

La institución de las promesas en las sociedades humanas es un claro ejemplo de cómo funciona una ética basada en lo que nos debemos los unos a los otros. El ejemplo es de Kant: si yo prometo a alguien que llevaré a cabo una determinada acción, pero desde el comienzo he tomado la decisión de 
que no la llevaré a cabo, de que defraudaré mi promesa con el fin de obtener ciertos beneficios que supuestamente superan desde el punto de vista utilitario el quedar ante los demás como alguien que no cumple lo que promete, estoy negando la posibilidad de que semejante estrategia (que no tiene en cuenta lo que nos debemos los unos a los otros) siga funcionando en mi beneficio; tarde que temprano nadie volverá a creer en mis promesas $\mathrm{y}$, por tanto, los supuestos beneficios que obtenía prometiendo para no cumplir desaparecerán por completo. Kant va aún más allá generalizando esta idea a partir de su concepto del imperativo categórico: todos deberíamos obrar como si el lema o máxima con el que pudiera describirse nuestra acción pudiera convertirse en una ley universal. Si yo prometo que devolveré un bien que me ha sido dado en préstamo pero tengo la intención, supuestamente astuta, de no devolverlo y guardarlo para mi usufructo, debería preguntarme si el lema de mi acción ("prometer falsamente me permitirá usufructuar un bien que no me pertenece") podría convertirse en una ley universal para mí y para los demás. Si lo miro desde esa perspectiva mi acción no tiene nada de astuta: nadie me daría nada en préstamo porque de antemano se sabría que mis promesas son falsas y nadie prestaría nada a nadie porque resultaría obvio que el único objetivo de las promesas es engañar a quien realiza el préstamo para que pierda el usufructo del bien, con lo que, desaparecida la institución de las promesas en las sociedades humanas, tanto yo como los demás terminaríamos perdiendo (18).

En el campo de la producción y la difusión del conocimiento las publicaciones científicas periódicas constituyen una de esas instituciones de las sociedades humanas en las que, como en la institución de las promesas, la ética de lo que nos debemos los unos a los otros resulta primordial. Las publicaciones biomédicas no son una excepción; todo lo contrario, en ellas, en la medida en que se están difundiendo conocimientos sobre lo que acontece con seres humanos en situaciones de salud o enfermedad o sobre lo que acontece con seres vivientes que atañen directa o indirectamente a la salud o enfermedad de seres humanos, las consideraciones éticas alcanzan su máximo valor, sin que por ello se pueda afirmar que en otro tipo de publicaciones periódicas científicas dichas consideraciones puedan ser mas relajadas. En ningún caso. La producción del conocimiento, el desarrollo de las ciencias y la difusión de los nuevos conocimientos se basan, como las promesas, en la confianza mutua entre quienes producen los conocimientos, quienes financian dicha producción, quienes difunden dichos conocimientos y quienes los consumen, de que a todo lo largo del proceso no se ha dado ningún paso en el que uno solo de los actores hubiese intentado obtener una ventaja fraudulenta para sí o para su grupo olvidando lo que nos debemos los unos a los otros. Retomando a Kant y su imperativo categórico: si en la base de la institución de la producción y difusión del conocimiento hubiese con demasiada frecuencia una promesa incumplida y lo que recibe el consumidor de conocimiento e información no es conocimiento científico sino otra cosa (investigaciones fraudulentas por parte del investigador que quiere sacar ventajas de su posición, publicidad disfrazada de conocimiento científico por parte de quienes financian la investigación porque quieren situar mejor un producto en el mercado, publicaciones acomodadas por parte de las publicaciones biomédicas con cualquier intención: mejorar su nivel de impacto, vender publicidad, evitar que alguien tenga la primacía en la publicación de una investigación en determinado campo, etc.), desaparecería por completo la credibilidad de las publicaciones científicas periódicas y la ciencia sufriría con ello enormemente mientras se inventa otra manera, si es que existe, de difundir el conocimiento que produce.

Como se comprenderá a partir del enfoque que he realizado, en los trabajos anteriores de este suplemento se han discutido con suficiente profundidad la mayoría de los conflictos éticos que pueden surgir en el proceso de producir conocimiento y difundirlo.

En la Historia de Acta Médica Colombiana el Dr. Paulo Emilio Archila ha logrado plasmar el desarrollo histórico de una revista que, como la nuestra, ha alcanzado su plena madurez y desarrollo manteniendo estándares éticos y editoriales que nada tienen que envidiarle a las publicaciones científicas periódicas de países plenamente desarrollados, en un país que, como el nuestro, no forma parte de ese selecto grupo de países.

En las Normas de Publicación en Revistas Biomédicas el Dr. Eduardo Zúñiga nos ha mostrado de qué manera, hasta en el asunto aparentemente tan sencillo de definir quién es y quién no es el autor de un trabajo de investigación, surgen conflictos que sólo pueden ser evitados desde la ética de lo que nos debemos los unos a los otros, conflictos que dan paso a los preceptos normativos y a las leyes sólo cuando no fue posible resolver el conflicto de autoría cuando apenas surgía.

En el trabajo Cómo Citar Referencias Bibliográficas del Dr. Roberto D'Achiardi, en apariencia sólo técnico, se esconde el fantasma del plagio y de qué manera evitarlo citando siempre de la manera más precisa las ideas de los demás.

En las Guías Mínimas para Reportar Estudios Aleatorizados (CONSORT) y Revisiones Sistemáticas y Metaanálisis (PRISMA), también en apariencia tan técnico, el Dr. Rodolfo Dennis nos pone a pensar sobre la financiación del proceso y los posibles sesgos que afectan los resultados de los estudios aleatorizados o la evidencia acumulativa de las revisiones sistemáticas y metaanálisis, desde el trabajo de los investigadores hasta las herramientas utilizadas.

Finamente, el trabajo de la Dra. María Nelly Niño de Arboleda sobre Conflicto de Intereses en Publicaciones Biomédicas es, sin lugar a dudas, un trabajo sobre los conflictos éticos que pueden surgir en este campo y la manera de resolverlos.

Sólo me queda insistir en que la mejor manera de resolver los conflictos éticos que surgen alrededor de las publicaciones biomédicas es evitarlos. Bastaría obrar bien, en el 
sentido de obrar siempre pensando en los demás (en lo que nos debemos los unos a los otros), para que dichos conflictos no surgieran. Sin embargo, cuando surgen, la mejor manera de resolverlos es apelando a la transparencia: cuando todo ha sido claro, cuando las fuentes de financiación se han establecido formalmente, cuando el papel de cada autor o posible autor ha sido definido, cuando se ha declarado si el trabajo ha sido publicado previamente en todo o en parte, cuando se han protegido los derechos de los participantes en la investigación y se esclarece en ella de qué manera y cuándo una publicación científica periódica, como Acta Médica Colombiana, tiene claras políticas, explícitas, sobre el sistema de revisión por pares evaluadores, la declaración de conflictos de interés y la posibilidad de discutir en un ambiente académico mediante, por ejemplo, cartas a los lectores, los hallazgos de una determinada investigación, resulta fácil dirimir cualquier conflicto (19).

El Committee on Publication Ethics (COPE) ha hecho público unos Principles of Transparency and Best Practice in Scholarly Publishing (20) (Principios de Transparencia y Buena Práctica en Publicaciones Académicas), avalados por la World Association of Medical Editors (WAME), el Directory of Open Access Journals (DOAJ), y la Open Access Scholarly Publishers Association (OASPA), con directrices muy sencillas y claras sobre las bases requeridas por cualquier publicación científica periódica para la resolución de conflictos éticos. Acta Médica Colombiana adhiere plenamente a dichos principios.

\section{Referencias}

1. Real Academia Española. Ética. En Diccionario de la Lengua Española (23 edición). Bogotá: Editorial Planeta Colombiana; 2014.

2. Corominas J, Pascual JA. Diccionario Crítico Etimológico Castellano e His- pánico. Volumen II. Madrid: Editorial Gredos; 1980: pp 818-819.

3. Aristotle. Nicomachean Ethics. 1103a-1103b. Barttlet RC, Collins SD (Translators). Chicago: The University of Chicago Press; 2011. Edición en formato digital: archivo Kindle.

4. Matijasevic E. Aliviar siempre. Acta Med Colomb 2011; 36 (1): 4-9.

5. Guariglia O, Vidiella G. Breviario de Ética. Buenos Aires; Edhasa; 2011: pp 23-25.

6. Real Academia Española. Moral. En Diccionario de la Lengua Española (23 edición). Bogotá: Editorial Planeta Colombiana; 2014.

7. Corominas J, Pascual JA. Diccionario Crítico Etimológico Castellano e Hispánico. Volumen IV. Madrid: Editorial Gredos; 1980: pp 136-137.

8. Scanlon T. What We Owe to Each Other. Cambridge, Massachusetts: Belknap Press; 2000: 432 pp.

9. Du Roy O. La règle d>or, histoire d > une maxime morale universelle : Volume 1, De Confucius à la fin du XIXe siècle. Paris: CERF; 2012: 901 pp.

10. Confucius. Analects. 15:24. Annping Chin (Translator). New York: Penguin Books; 2014. Edición en formato digital: archivo Kindle.

11. Confucius. Analects. 4:15. Annping Chin (Translator). New York: Penguin Books; 2014. Edición en formato digital: archivo Kindle.

12. Mahabarata. Shanti. Moksha Dharma Parva. 12:86:251. Bibek Debroy (Translator). Volume 9. Haryana, India: Penguin Books; 2015. Edición en formato digital: archivo Kindle.

13. Shaw GB. Maxims for Revolutionists. [Internet]. Consultado el 20 de junio de 2015. Disponible en http://archive.org/stream/maximsforrevolut26107gut/26107. $\underline{\mathrm{txt}}$

14. Mateo. 22:39. Biblia De Jerusalén. Cuarta Edición. Bilbao: Editorial Desclée De Browver, 2009. Edición en formato digital: archivo Ibooks.

15. Marcos. 12:31. Biblia De Jerusalén. Cuarta Edición. Bilbao: Editorial Desclée De Browver, 2009. Edición en formato digital: archivo Ibooks.

16. Lucas. 10:27. Biblia De Jerusalén. Cuarta Edición. Bilbao: Editorial Desclée De Browver, 2009. Edición en formato digital: archivo Ibooks.

17. Camps V. Una vida de calidad: Reflexiones sobre la Bioética. Barcelona: Editorial Crítica; 2002: pp 127-128.

18. Kant E. Fundamentación de la metafísica de las costumbres. 4:402-4:403. GarciaMorente M (Traductor). Madrid: Espasa-Calpe SA; 1980: pp 15-17.

19. Graf C, Wagner E, Bowman A, et al. Best Practice Guidelines on Publication Ethics: a Publisher's Perspective. Int J Clin Pract 2007; 61 (Suppl. 152): 1-26.

20. Committee on Publication Ethics (COPE). Principles of Transparency and Best Practice in Scholarly Publishing. Consolidate el 20 de junio de 2015. Disponible en: http://publicationethics.org/files/Principles of Transparency and Best Practice in Scholarly Publishingv2.pdf 